\title{
6th DACH+ Conference on Energy informatics (EnInf 2017)
}

\author{
Silvia Santini ${ }^{1}$ - Verena Tiefenbeck ${ }^{2}$
}

(C) Springer-Verlag GmbH Germany 2017

This volume contains the scientific contributions accepted for presentation at the 6th DACH+ Conference on Energy Informatics (EnInf 2017). The objective of the DACH+ conference series on Energy Informatics is to promote the research, development, and implementation of information and communication technologies in the energy domain and to foster the exchange between academia, industry, and service providers across Germany (D), Austria (A) and Switzerland $(\mathrm{CH})$ and their neighboring countries (DACH+). The Energy Informatics conference series is a joint initiative of the Swiss Federal Office of Energy, the Austrian Ministry for Transport, Innovation and Technology, and the German Federal Ministry for Economic Affairs and Energy and is also supported by the German Informatics Society, the Austrian Computer Society, and the Swiss Informatics Society.

The 6th edition of the conference took place in Lugano, Switzerland, on October 5-6, 2017 at the Università della Svizzera italiana (USI). More than 90 participants from six different European countries as well as the USA attended the event. This makes the 2017 edition one of the most successful in terms of number of attendees and diversity. The conference provided a forum for very vivid discussion between researchers and also offered the opportunity to exchange ideas and experiences with several representatives from industry. As in the previous years, the conference was accompanied by a Ph.D. workshop organized by Prof. Sebastian Lehnhoff and Dr. Astrid Niesse, which took place the day before the conference. We would like to thank the

Silvia Santini

silvia.santini@usi.ch

1 Università della Svizzera italiana (USI), Lugano, Switzerland

2 ETH Zürich, Zürich, Switzerland organizers, participants and all shepherds of the Ph.D. workshop for their contribution to the conference.

With a total of 62 paper submissions, the 6th edition of the DACH+ Conference on Energy Informatics recorded the highest number of submissions since the inception of the conference series. The articles submitted were reviewed and discussed by a panel of 25 experts who accepted to be part of the conference's Program Committee. In some cases, external reviewers were asked to contribute to the evaluation of the papers. We sincerely thank all the members of the Program Committee as well as the external reviewers for their invaluable work.

This volume contains the 15 full papers describing mature research contributions and the eight research-in-progress papers presented at the conference. Further, the volume includes the extended abstracts of the 13 posters and five demos that have been accepted for presentation at the conference. We sincerely thank the Poster and Demo Chairs of the conference, Dr. Vlad Coroama and Dr. Slobodan Lukovic, who selected the poster and demo abstracts collected in this volume.

This journal issue reflects the large variety of topics that is characteristic for the Energy Informatics field. Contributions cover the entire value chain from energy supply (Hartmann et al.) over distribution (Ascher and Bytschkow) and storage (Steber et al.). This year's conference had a strong focus on diverse demand-side management topics (e.g., Barth and Wagner, Lübkert et al., Ludwig et al.). Application areas include e-mobility (Buffat et al., Knirsch et al., Schmidt et al.), and residential and commercial buildings (Rominger et al., Zemtsov et al.). Many disciplines contribute to these research endeavors, including work rooted in market design (Bagemihl et al., Hunziker et al., Mengelkamp et al.), simulation studies (Exel and Frey, Marten et al., Steinbrink et al.), machine learning and clustering (Becker and Kleiminger, 
Sangogboye and Kjærgaard, Schöb et al., Zehetbauer et al.), standardization (Razik et al.) and protocol security (Bao et al.) -all with a clear reference to energy applications enabled by information and communication technologies.

Out of 23 papers presented at the conference, three were nominated as candidates for the Best Paper Award. The winning paper is by Barth et al. (\#2), the other two nominees were by Buffat et al. (\#4), and Schöb et al. (\#12). We would like to thank the members of the Best Paper Award Committee-Prof. Hartmut Schmeck, Prof. Miroslaw Malek and Prof. Dominik Engel—for the additional time and work they invested in selecting the final winner of the conference's Best Paper Award.

The successful organization of the 6th DACH+ Conference on Energy Informatics has been possible only thanks to the hard, volunteer work of members of our research community, who deserve our deepest gratitude. Aside from those we already mentioned, we would like to explicitly thank all members of the DACH+ Energy Informatics Steering Committee as well as the other members of the Organizing Committee: Liliane Ableitner (Publication Chair), Anselma Wörner (Publicity Chair), Elisa Larghi (Registration Chair), and Elena Di Lascio (Local Arrangement Chair).

We wish you a pleasant and inspiring read through the articles collected in this volume!

General Chair

Silvia Santini

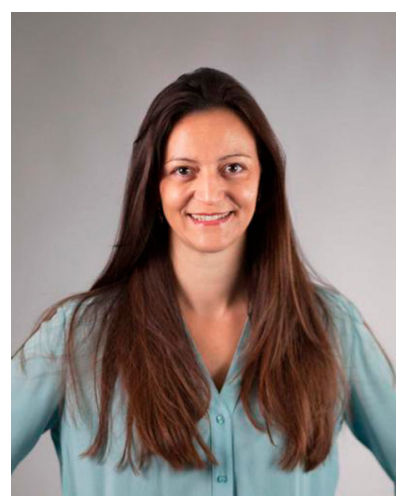

IMWUT and a member of the Steering Committee of the ACM UbiComp and DACH+ Energy Informatics conference series.

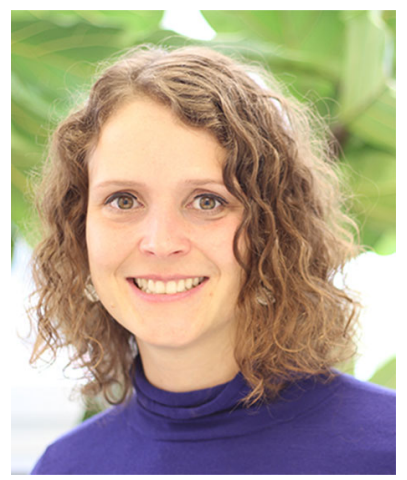

Verena Tiefenbeck is a senior research assistant at ETH Zurich, Switzerland. She works as the chair of Information Management, where she leads the Bits to Energy Lab at ETH Zurich, an interdisciplinary team that combines digital technologies and behavioral research to foster resource conservation. She completed her PhD in 2014 under the supervision of Prof. Elgar Fleisch at ETH Zurich. Prior to that, she spent 3.5 years in Boston as visiting $\mathrm{PhD}$ student at the Massachusetts Institute of Technology and as research assistant at the Fraunhofer Center for Sustainable Energy Systems. Verena Tiefenbeck holds diplomas (M.Sc. equiv.) in Mechanical Engineering and Management from both TU Munich and from Ecole Centrale Paris (TIME double degree program). Verena Tiefenbeck focuses on empirical quantitative research that combines information and communication technologies with methods and theories from social science research, in particular from behavioral economics.
Friedemann Mattern's Distributed Systems Group at the Department of Computer Science of ETH Zurich, Switzerland. From November 2010 until February 2011 she joined Leonidas Guibas's research group at Stanford University as a visiting scholar. Silvia completed her PhD Thesis under the supervision of Prof. Friedemann Mattern at ETH Zurich in 2009 and graduated in Telecommunication Engineering (with honors) from the Sapienza University of Rome, Italy, in 2004. Silvia Santini's research focus is on mobile sensing and low-power networks and encompasses other topics in cyber-physical systems, ubiquitous computing, and Internet of things. She has served as the member of the Organizing and Program Committees of several leading conferences, including UbiComp, SenSys, BuildSys, e-Energy, EnInf, PerCom, MobiSys, IPSN, and INFOCOM. She is an Editor of PACM
Silvia Santini is an Associate Professor at the Faculty sità della Svizzera italiana (USI) in Lugano, Switzerland. Before ciate Professor position at TU Dresden, Germany, where she and an Assistant Professor posiat TU Darmstadt, Germany, where she led the Wireless Sensor Networks Lab. Previously, from 2009 until 2011, she was a from 2009 until 2011, she was a

.

